\title{
Can Gravitation really be absorbed into the Frame of Space and Time?
}

\section{By Sir Joseph LaRmor, F.R.S. ${ }^{1}$}

$\mathrm{A} \mathrm{N}$ answer to this question in the negative has been advanced in a previous paper on the gravitational deflection of light (Phil. Mag., Jan.). The destructive paradoxes concerned with the recent gravitation theory, which were unfolded by M. Jean Le Roux, professor at Rennes, in three notes in the Comptes rendus (Nov. 6, Dec. 4 and 22), after that paper was completed, were referred to in a footnote in support of this departure from the familiar answer. These objections require to be further considered; for at first sight they are destructive to all such theories, including the modification there substituted. If an orbit is postulated to be a curve of minimal length in a fourfold expanse of space-time, the element of length (or distance-interval) must be expressed for it locally, and can involve as variables only its own co-ordinates and their differentials. Yet in the cases that have been worked out, the element as determined involves also the concurrent coordinates of the other interacting masses; with tall these variables present, it could not belong to a curke in a fourfold at all. This destructive dilemma applies very widely.

There may be a suggestion to evade it, in the theory as modified into one of dynamical Action, along the line (already indicated by A. A. Robb) that the idea of distance cannot subsist in the pseudospace at all. For within an infinitesimal fourfold spherical domain with radius a very small interval $d s$, the co-ordinates would have an infinite range of values. The idea of locality, essential to real space, is thus absent. The fourfold expanse could still be utilised to express conveniently the domains of integration : but where distances have to enter they must be in threefold real space, though it can be variable and be associated with time also variable. Such real spaces and times would be locally not unique; they constitute a Lorentz group of interchangeable forms. The modified gravitational scheme of the previous paper, with its reduction of the influences on radiation to one-half of the accepted values, might, merely by avoiding the idea of fourfold interval interpreted as a geometric distance, possibly still manage to evolve as a dynamical formulation.

But this train of ideas need not be pursued ; for in fact the criticism, which seems destructive of a quasi-

${ }^{1}$ Abstract of a paper read on January 22 at the Cambridge Philosophical Society. geometric scheme for gravitation, does not inhere at all in the dynamical domain of Action. The type of procedure for minimising the total Action, when more closely exhibited, would run in principle as follows. Assume some approximate specification for the orbital paths in the fourfold, close of course to the Newtonian. solution. The orbits thus assumed will determine the nature of the fourfold space-time expanse (namely $d s^{2}$ ) already adjusted to minimal Action, in which they exist. For each such specification calculate the density of Action in this fourfold expanse, after the manner of approximate modifications as developed by Einstein; and thence find by integration the total Action of the system corresponding to these assumed orbital forms. The forms of the orbits would enter in the expression for the linear element $d s$ defining the space determined by these orbits and necessarily containing them. By taking varied forms of the orbits, different forms of $d s$ and different values of the total Action would be obtained. The aim would be to adapt the forms of the orbits so that the Action thus determined from them should remain stationary for all slight variations. The way to carry this out would be to minimise the Action further for joint variation of all the orbits, exactly on the lines of the previous paper. The space itself, being determined by the orbits, also changes as the orbits are varied; and it is not at all involved that $d s$ remains the elemental distance in the same space throughout the procedure.

It would appear then that the Minkowskian method of fourfold spatial analysis as generalised by Einstein for adaptation of gravitation into the optical and electrodynamic group of frames, can be saved from the destructive criticism of $M$. Le Roux. But to this end the postulate of absorption of gravitation into the spatial frame must be abandoned; and the principle of equivalence of gravitation and acceleration would disappear. The application of the mathematical spatial analysis to astronomy and optics would be reconstructed as a dynamical theory of normal type, unfolding itself in terms of a distribution of Action located ultimately throughout the region of the problem : but the results as modified would still require actual confirmation. If, however, any gravitational influence on light is finally established by the astronomical observations, this type of analysis by aid of a varying spatial frame may remain the most effective way to include it in theory.

\section{The Nature of Gels.}

\section{By Dr. S. C. Bradford.}

I $\mathrm{T}$ has been known, probably from the earliest times, that when sufficiently concentrated solutions of certain substances, such as gelatin and agaragar, are allowed to cool, instead of depositing crystals of the dissolved substance, the whole liquid turns into a jelly. It is natural, therefore, that speculations on the nature of jellies should have been rife long before Graham, in I86I, first pointed out the slow rate of diffusion of colloid substances which distinguished them from bodies which separate from solution in the ordinary crystalline form.

The many theories of gel structure fall naturally under three heads: (I) One-phase or molecular systems, (2) two-phase liquid-liquid systems, and (3) two-phase liquid-solid systems. To the first class belongs Proctor's hypothesis that a gel is a more or less solid solution of a liquid in the colloid substance, in which both constituents are within the range of molecular attractions. This view is very similar to the "super-cooled liquid" theory of glass, and, like that, has difficulty in explaining the loss of mobility which occurs on setting. Proctor suggests that the transformation consists in the formation of tenuous crystals, which interlace and possibly anastomose. Later experiments ${ }^{\mathbf{1}}$ show that gelation is really an extreme case of crystallisation, but this suggestion would bring Proctor's theory into the third class. In either case, however, his experiments are important, as they show that the swelling of gelatin in

1 Bradford, Science Progress, I917, I2, 62; Biochem. Jour. 1918, I2 357; I920, I4, 9I; I92 I, I5, 553; and "The Physics and Chemistry of Colloids," Discussion by the Faraday Society, etc., London, I 921. 